\title{
Correction to: Validation of estimated glomerular filtration rate equations for Japanese children
}

\author{
Yoshimitsu Gotoh ${ }^{1}$ (1) Osamu Uemura ${ }^{2} \cdot$ Kenji Ishikura $^{3} \cdot$ Tomoyuki Sakai $^{4} \cdot$ Yuko Hamasaki $^{5} \cdot$ Yoshinori Araki $^{6}$. \\ Riku Hamada ${ }^{7}$. Masataka Honda ${ }^{7}$. On behalf of the Pediatric CKD Study Group in Japan in conjunction with the \\ Committee of Measures for Pediatric CKD of the Japanese Society of Pediatric Nephrology
}

Published online: 24 August 2018

(c) Japanese Society of Nephrology 2018

\section{Correction to: \\ Clinical and Experimental Nephrology \\ (2018) 22:931-937 \\ https://doi.org/10.1007/s10157-018-1529-7}

In the original publication, the author name was published incorrectly as "Riku Hamda". The corrected name is given in this Correction.

Yoshimitsu Gotoh

ygotoh@nagoya2.jrc.or.jp

1 Department of Pediatric Nephrology, Japanese Red Cross Nagoya Daini Hospital, 2-9 Myoken-cho Showa-ku, Nagoya, Aichi 466-8650, Japan

2 Department of Clinical Medicine, Japanese Red Cross Toyota College of Nursing, 12-33 Nanamagari, Hakusan-cho, Toyota, Aichi 471-8565, Japan

3 Department of Nephrology and Rheumatology, National Center for Child Health and Development, 2-10-1 Okura, Setagaya-ku, Tokyo 157-8535, Japan
4 Department of Pediatrics, Shiga University of Medical Science, Seta, Ootsu, Shiga 520-2192, Japan

5 Department of Pediatric Nephrology, Toho University Faculty of Medicine, 6-11-1 Oomori Nishi Seta, Ootsu, Shiga 143-8541, Japan

6 Department of Pediatrics, Hokkaido Medical Center, 5-7-1-1 Yamanote 5jyo Nishi-ku, Sapporo, Hokkaido 063-0005, Japan

7 Department of Nephrology, Tokyo Metropolitan Children's Medical Center, 2-8-29 Musasidai, Futyu-shi, Tokyo, Tokyo 183-8561, Japan 